\title{
Flore et végétation de la Réserve Spéciale de Faune de Gueumbeul (Sénégal)
}

\author{
Babacar FAYE ${ }^{1 *}$, César BASSENE ${ }^{1,2}$, Abdou Aziz CAMARA ${ }^{1}$, Ablaye NGOM$^{1}$, \\ Mame Samba MBAYE ${ }^{1}$ et Kandioura NOBA ${ }^{1}$ \\ ${ }^{1}$ Laboratoire de Botanique et Biodiversité, Département de Biologie Végétale, Faculté des Sciences et \\ Techniques, Université Cheikh Anta Diop. BP 5005 Dakar-Fann, Sénégal. \\ ${ }^{2}$ Section Productions Végétales et Agronomie, UFR des Sciences Agronomiques, de l'Aquaculture et des \\ Technologies Alimentaires, Université Gaston Berger de Saint-Louis, Sénégal. \\ *Auteur correspondant; E-mail: fayebab@live.fr; Tel: (+221) 774515026
}

\section{REMERCIEMENTS}

Les auteurs remercient la Direction des Parcs Nationaux (DPN) qui, à travers l'assistance financière, a facilité la réalisation de ce travail.

\section{RESUME}

L'étude a été réalisée entre 2010 et 2014 dans la Réserve Spéciale de Faune de Gueumbeul (RSFG) qui est une zone humide classée site Ramsar et abritant une faune riche et variée ainsi qu'une flore diversifiée mais peu connue. Cette étude est entreprise pour déterminer la structure de la flore et de la végétation, élément indispensable pour une bonne gestion des ressources de cette réserve. Elle a été réalisée par la méthode des relevés phytosociologiques. Ces travaux ont permis de répertorier 100 espèces réparties dans 82 genres et 42 familles. Il ressort de l'analyse des types biologiques que la réserve est composée essentiellement d'herbacées à dominance annuelle $(60 \%)$. Cependant les espèces pérennes représentent une part importante $(40 \%)$ de cette flore. Sur le plan de la répartition géographique, les résultats montrent que les espèces d'affinités afroasiatiques (30\%) et africaines (28\%) sont les plus représentatives de la flore suivies des espèces pantropicales (12\%) puis des espèces afro-asiatiques et australiennes (8\%). La végétation de la réserve est caractérisée par une dominance des espèces rares ou accidentelles (78\%) suivies des espèces accessoires (19\%) dont Opuntia tuna tandis que les espèces fréquentes ne représentent que $3 \%$ de la flore globale. Les résultats de cette étude révèlent cinq (5) groupements végétaux dont deux halophytes et trois non halophytes établis sur les dunes ou les plaines basses. Cette étude permet une meilleure connaissance de la flore et de la végétation de la RSFG afin de mieux gérer ces ressources végétales.

(C) 2018 International Formulae Group. All rights reserved.

Mots clés: Flore, végétation, Réserve spéciale de faune, Gueumbeul, Sénégal.

\section{Flora and vegetation of the Gueumbeul Wildlife Reserve (Senegal)}

\begin{abstract}
The study was carried out between 2010 and 2014 in the Gueumbeul Wildlife Reserve (RSFG) which is a wetland classified as a Ramsar site and home to a rich and varied fauna as well as a lesser known diverse flora. This study is an undertaking to determine the structure of the flora and vegetation, which are essential for
\end{abstract}


the proper management of the resources of this reserve. It was carried out using the phytosociological survey method. This work allowed to list 100 species distributed in 82 genera and 42 families. The analysis of biological types shows that the reserve is composed mainly of herbaceous plants with an annual predominance (60\%). However, perennial species constitute an important part (40\%) of this flora. In terms of geographical distribution, the results show that Afro-Asia (30\%) and African (28\%) affinity species are the most representative of flora followed by pantropical species (12\%) and Afro-Asian and Australian (8\%). Reserve vegetation is dominated by rare or accidental species (78\%) followed by catch species (19\%), including Opuntia tuna and frequent species (3\%). The results of this study revealed five (5) plant groups, including two halophytes and three non-halophytes established on dunes or low plains. This study allows a better knowledge of the flora and the vegetation of the RSFG in order to better manage the vegetal resources.

(C) 2018 International Formulae Group. All rights reserved.

Keywords: Flora, vegetation, Special reserve of wildlife, Gueumbeul, Senegal.

\section{INTRODUCTION}

La flore phanérogamique du Sénégal est caractérisée par des espèces herbacées qui en constituent plus de 50\%. Ces herbacées sont généralement annuelles ce qui leur permet de s'adapter aux conditions mésologiques sans cesse changeantes. La savane et la steppe sont globalement les formations végétales dominantes ( $\mathrm{Ba}$ et Noba, 2001). Les efforts consentis par le Sénégal en matière de conservation de la biodiversité ont abouti à la création d'un réseau constitué de parcs nationaux et de réserves de faune (WWF WAMER, 2011). Parmi ces réserves, nous notons la réserve spéciale de faune de Gueumbeul qui appartient à ce réseau géré par la direction des parcs nationaux (DPN). Ces aires protégées sont un instrument crucial du développement durable de par leur rôle dans le maintien des services écosystémiques (UICN, 2014). Aujourd'hui, outre leur valeur de conservation, ces aires protégées offrent une série de biens et de services. Ces biens et services sont la diversité biologique, l'écotourisme, les activités récréatives et les moyens de subsistance des communautés locales (CDB, 2004). Les aires protégées servent aussi de refuge à de nombreuses espèces et constituent un patrimoine exceptionnel pour les populations locales (Abdourhamane et al., 2013). Cependant, l'utilisation excessive de ces ressources combinée aux aléas climatiques entraîne une régression progressive de la biodiversité (Bassène et al., 2014).

La Réserve Spéciale de Faune de Gueumbeul (RSFG) est une zone humide d'importance internationale classée site Ramsar depuis le 26 septembre 1986. Elle sert de centre de multiplication en semi captivité pour le redéploiement de la faune sahélienne dans leurs milieux d'origine (Diop et Mandiang, 2001).

Elle abrite une faune riche et variée répartie en trois types : la faune autochtone, la faune introduite et la faune en migration (DPN, 2010). La faune autochtone comprend essentiellement des singes rouges, des phacochères, des lièvres, des écureuils et celle introduite des gazelles et des oryxs. Quant à la faune en migration, elle est principalement composée d'oiseaux. En effet, des milliers d'oiseaux migrateurs séjournent chaque année au niveau de la cuvette de Gueumbeul (Thiam, 2004).

La flore de la RSFG est également diversifiée. La végétation est de type sahélien à prédominance d'épineux (WWF WAMER, 2011). Ces essences végétales constituent pour la faune divers habitats et d'importantes réserves fourragères (Anonyme, 2009). De nos jours, une étude complète n'a pas encore été faite sur la flore et la végétation de la RSFG. Or, une bonne connaissance des ressources naturelles de la réserve, notamment 
de la faune suppose une connaissance approfondie de la flore et de la végétation.

Ce travail envisage d'étudier la flore et la végétation de la RSFG et se propose de déterminer la composition de la flore, son spectre taxonomique, biologique et la répartition géographique des espèces puis les caractéristiques de la végétation et en particulier les indices de fréquence ainsi que les différents groupements végétaux.

\section{MATERIEL ET METHODES}

\section{Présentation du site d'étude}

La Réserve Spéciale de Faune de Gueumbeul a été créée par le décret n ${ }^{\circ} 83-550$ du 30 mai 1983. Elle se trouve dans le delta du fleuve Sénégal (Figure 1). Elle est située à $7 \mathrm{~km}$ au sud de la commune de Saint-Louis sur la route de Gandiole. Elle se trouve dans une dépression (cuvette) peu profonde et couvre une superficie totale de 720 ha. La réserve se présente sous forme d'une cuvette d'eau saumâtre entourée d'une végétation clairsemée.

\section{Méthodologie}

Les études ont été effectuées entre octobre 2010 et septembre 2014. Pour déterminer la structure de la flore et de la végétation de la Réserve spéciale de faune de Gueumbeul, le travail a consisté à faire des inventaires floristiques et des relevés phytosociologiques selon l'approche sigmatiste (Meddour, 2011). Les relevés phytosociologiques ont été réalisés dans des surfaces floristiquement homogènes au moins égales à celle de l'aire minimale. Pour l'étude de la végétation ligneuse sahélienne, l'aire minimale est de $2500 \mathrm{~m}^{2}$ (Boudet, 1984 cité par Akpo et Grouzis, 1996). Quant à l'étude de la strate herbacée, cette aire est de $64 \mathrm{~m}^{2}$ (Diop et al., 2008).

Les identifications des espèces ont été faites à l'aide de la flore de Berhaut (1967) et par comparaison avec des échantillons déposés dans les Herbiers DAKAR et IFAN (Université Cheikh Anta Diop).

La nomenclature utilisée est celle de Lebrun et Stork (1991, 1992, 1995, 1997). La classification de Raunkier (1934), adaptée à la zone tropicale ou la saison défavorable correspond à la saison sèche (Trochain, 1966 ; Lebrun, 1966), a été utilisée afin de déterminer les types biologiques des espèces. Cette classification distingue les 6 formes biologiques suivantes: les Phanérophytes $(\mathrm{P})$, les Chaméphytes (C), les Hémi cryptophytes $(\mathrm{H})$, les Géophytes (G), les Thérophytes $(\mathrm{T})$ et les Parasites (Par). En ce qui concerne les répartitions géographiques des espèces, les informations proviennent essentiellement des flores de Hutchinson et Dalziel (1954, 1963, 1968). L'abondance dominance moyenne a été obtenue en rendant quantitatif le codesemi quantitatif d'abondance-dominance de Braun Blanquet (Gillet, 2000) et en faisant la moyenne des abondances dominances suivant cette formule.

$$
\mathrm{ADm}=\sum \mathrm{AD}
$$

Avec ADm: Abondance dominance moyenne et $\mathrm{AD}$ : Abondance dominance

La fréquence relative d'une espèce a été calculée en établissant le rapport entre le nombre de relevés où l'espèce est présente sur le nombre total de relevés suivant cette formule.

$$
\mathrm{Fr}=\mathrm{n} / \mathrm{N}
$$

Avec $\mathrm{n}$ représentant le nombre de relevés dans lesquels l'espèce est présente

$\mathrm{N}$ représente le nombre total de relevés

La qualification des espèces selon leur indice de fréquence a été définie par la méthode de Caratini (1985) (Tableau 1).

Les groupements végétaux sont déterminés à l'aide de l'analyse factorielle des correspondances (AFC). Elle a été appliquée à la matrice 45 relevés $x 100$ espèces au niveau de la RSFG. 


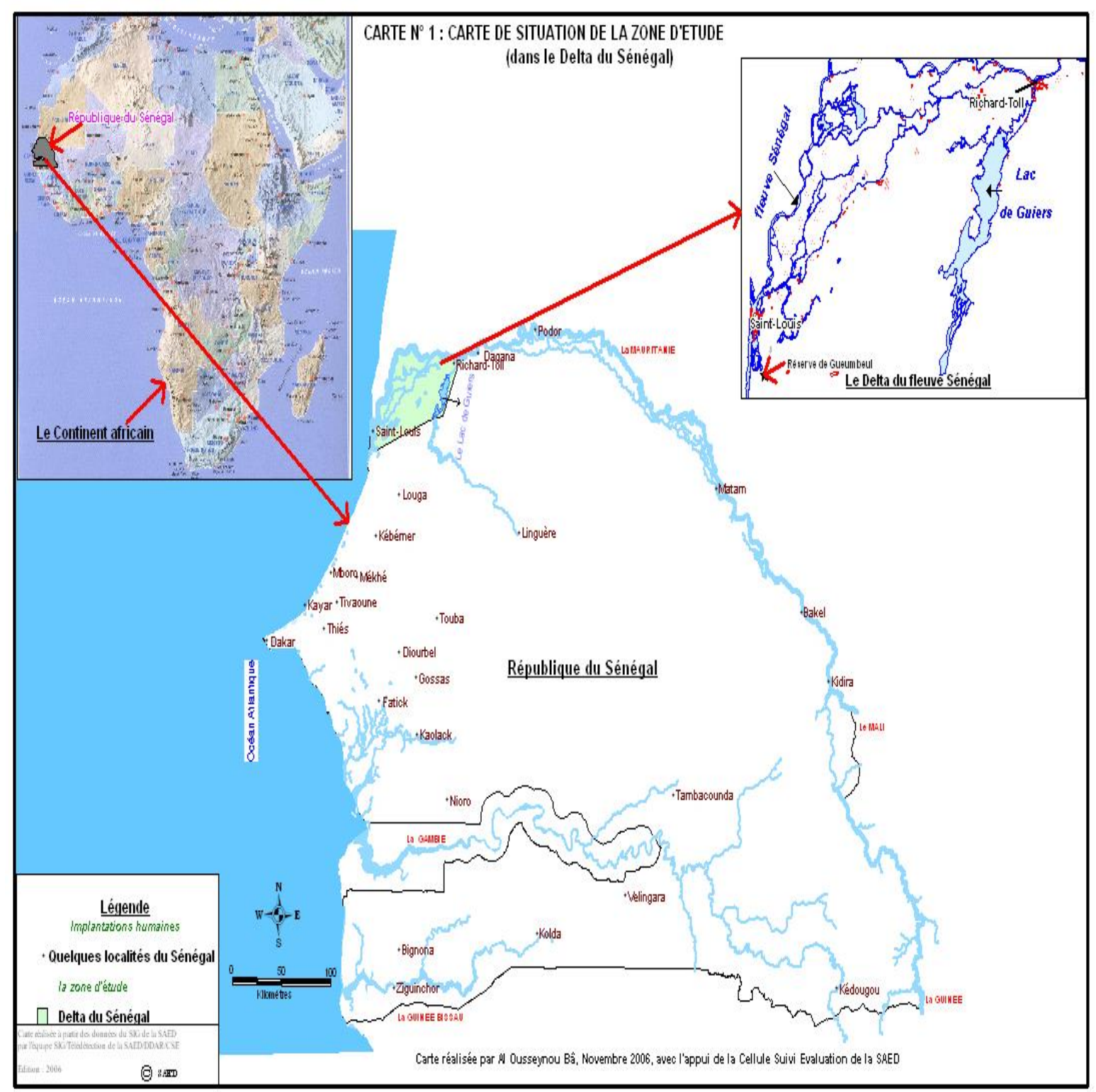

Figure 1: Localisation de la RSFG.

Tableau 1: Indices de Caratini.

\begin{tabular}{lcc}
\hline Fréquences & Indices & Qualification \\
\hline 0,8 à 1 & V & Constante \\
0,6 à 0,8 & IV & Abondante \\
0,4 à 0,6 & III & Fréquente \\
0,2 à 0,4 & II & Accessoire \\
0 à 0,2 & I & Rare ou Accidentelle \\
\hline
\end{tabular}




\section{RESULTATS}

\section{Etude de la flore}

Les résultats globaux de la flore et de la végétation de la RSFG sont consignés dans le Tableau 2. Ces résultats ont permis la détermination de la composition spécifique, $\mathrm{du}$ spectre taxonomique, biologique, de la répartition géographique ainsi que des indices de fréquence.

\section{Composition spécifique}

Les résultats de l'inventaire floristique de la Réserve Spéciale de Faune de Gueumbeul montrent que la flore est très diversifiée. Il ressort de ces résultats que cette flore est composée de 100 espèces réparties dans 82 genres et 42 familles (Tableau 2).

\section{Spectre taxonomique}

L'analyse du Tableau 3 révèle que toutes les espèces appartiennent au groupe des Angiospermes répartis dans deux classes que sont les Dicotylédones et les Monocotylédones. Les Poaceae et les Fabaceae sont les familles les plus représentées avec respectivement $18 \%$ et $17 \%$ des espèces. Les Poaceae et les Fabaceae regroupent avec les Convolvulaceae, les Amaranthaceae, les Aizoaceae, les Caryophyllaceae, les Cucurbitaceae et les Malvaceae, plus de la moitié des espèces (66\%) de la flore de la réserve spéciale de faune de Gueumbeul. Elles sont suivies des Acanthaceae, des Ampelidaceae, des Capparaceae, des Commelinaceae, des Cyperaceae, des Hyacinthaceae, des Nyctaginaceae, des Solanaceae et des Zygophyllaceae qui totalisent $43 \%$ des espèces. Les autres familles sont monospécifiques et sont représentées par les familles des Anacardiaceae, Apocynaceae, Araceae, Asparagaceae, Asteraceae, Avicenniaceae, Balanitaceae, Boraginaceae, Cactaceae, Chenopodiaceae, Combretaceae, Euphorbiaceae, Geraniaceae, Malvaceae, Pedaliaceae, Phyllanthaceae, Piperaceae, Polygalaceae, Rubiaceae, Salvadoraceae, Tamaricaceae et Verbenaceae qui regroupent $22 \%$ des espèces.

Il ressort aussi de l'analyse de ces résultats que la flore de la RSFG peut être divisée en trois groupes de familles:
- le premier groupe comprend les familles monospécifiques. Ce groupe représente $22 \%$ des espèces recensées soit plus de la moitié $(56,41 \%)$ des familles de la flore de la RSFG.

- le second groupe constitue les familles ayant un nombre d'espèces au moins égal à 2 . Il totalise $43 \%$ des espèces soit $38,46 \%$ des familles de la flore.

- le troisième groupe comprend les deux familles les plus diversifiées de la flore. Il s'agit des Poaceae et des Fabaceae qui comptent $35 \%$ des espèces et représentent $5,13 \%$ des familles.

La structure générale de la flore (Tableau 4) indique qu'elle est composée de $74 \%$ de Dicotylédones soit environ 3/4 de l'ensemble des espèces contre $26 \%$ de Monocotylédones soit environ $1 / 4$ des espèces.

\section{Spectre biologique}

La Figure 2 représente les résultats obtenus sur le spectre biologique de la flore de la Réserve Spéciale de Faune de Gueumbeul. Il ressort de ces résultats que $57 \%$ des espèces sont des Thérophytes. Ces Thérophytes constitués d'espèces annuelles sont essentiellement des Asteraceae des Poaceae, des Fabaceae, des Convolvulaceae et des Cucurbitaceae. Cependant, les Poaceae et les Fabaceae constituent l'essentiel de ces Thérophytes.

Les espèces pérennes représentent $43 \%$ de la flore. Elles sont composées de:

- Phanérophytes (20\%) appartenant principalement aux familles des Mimosaceae, des Cactaceae, des Rhizophoraceae, des Tamaricaceae et des Salvadoraceae.

- de Chaméphytes (11\%) constituées essentiellement, d'Amaranthaceae, d'Aizoaceae, et de Nyctaginaceae;

- Hémicryptophytes (6\%) constituées majoritairement de Poaceae et,

- Géophytes (6\%) constituées principalement de Liliaceae, Cyperaceae et Araceae.

\section{Répartitions géographiques}

Les résultats sur la répartition géographique des espèces de la flore de la RSFG sont consignés dans la Figure 3. Ces 
résultats montrent que $30 \%$ des espèces sont d'affinités afro-asiatiques, $28 \%$ africaines, $12 \%$ pantropicales, $8 \%$ afro-asiatiques et australiennes. Les espèces afro-malgaches représentent $4 \%$. Elles sont suivies des espèces afro-asiatiques et européennes, des afro-malgaches et asiatiques et des afroaméricaines avec chacun de ces groupes $3 \%$. Pour les espèces cosmopolites, afroeuropéennes, et les afro-asiatiques et américaines, chaque groupe représente $2 \%$ de la flore. Les espèces afro-américaines et européennes, les espèces afro-malgachesasiatiques-australiennes et européennes et les espèces afro-américaines-asiatiques et australiennes représentent $3 \%$ de la flore de la réserve.

\section{Etude de la végétation \\ Indices de fréquence}

Pour les indices de fréquence des espèces répertoriées dans cette réserve (Tableau 2), les espèces rares ou accidentelles sont les plus représentées (78\%). Elles sont suivies des espèces accessoires (19\%) et des espèces fréquentes $(3 \%)$. Les espèces constantes ou abondantes sont absentes. Les espèces rares ou accidentelles ne se rencontrent que dans $20 \%$ des relevés au plus. Parmi ces espèces nous pouvons citer: Acacia senegal, Acacia nilotica (FabaceaeMimosoideae), Adansonia digitata (FabaceaeBombacoideae), Sclerocarya birrea (Anacardiaceae), Combretum aculeatum (Combretaceae), Asparagus flagellaris (Asparagaceae), Zornia glochidiata, Sesbania pachycarpa, Indigofera tinctoria (FabaceaeFaboideae), Tribulus terrestris (Zygophyllaceae) Mitracarpus hirtus (Rubiaceae), Cressa cretica (Convolvulaceae), Senna italica (FabaceaeCaesalpinoideae). Les espèces accessoires représentent 19\%. Il s'agit de 19 espèces que sont: Opuntia tuna (Cactaceae) Acacia tortilis var. raddiana (Fabaceae), Achyranthes aspera (Amaranthaceae), Alysicapus ovalifolius, Indigofera aspera (Fabaceae), Aristida sieberiana, Dactyloctenium aegyptium, Cenchrus biflorus, Digitaria sp., Enteropogon prieurii (Poaceae) Merremia pinnata
(Convolvulaceae), Tetraena gaetula subsp. waterlotii (Zygophyllaceae), Corchorus tridens (Tiliaceae) Commicarpus helenae (Nyctaginaceae), Euphorbia balsamifera (Euphorbiaceae), Leptadenia lanceolata (Asclepiadaceae), Salsola baryosma, Salicornia senegalensis (Chenopodiaceae), Salvadora persica (Salvadoraceae). Quant aux espèces fréquentes, elles sont constituées de Prosopis glandulosa (FabaceaeMimosoideae), Commelina forskaolii (Commelinaceae) et Commiphora africana (Burseraceae).

\section{Identification des groupements}

L'analyse du Tableau 5 obtenu de l'analyse factorielle des correspondances montre que seul le premier axe réalise plus de 0,5 de valeur propre. La variance expliquée par les quatre premiers axes s'élève à $48,68 \%$. Les axes 1 et 2 totalisent $30,11 \%$ de cette variance soit $61,85 \%$ de la variance expliquée par ces quatre premiers axes. A lui seul, l'axe 1 présente $19,67 \%$ de variance. Le deuxième meilleur score est détenu par l'axe 2 d'où le choix porté sur les deux premiers axes. La moyenne des contributions des relevés à l'axe 1 est de 0,50 et celle des espèces 0,20 . Or, seuls les relevés et les espèces dont la contribution est supérieure à la moyenne des contributions à l'axe peuvent participer significativement. Par conséquent, peuvent contribuer significativement à l'axe des abscisses positives les relevés suivants: $\mathrm{R} 1, \mathrm{R}$ 3 et $\mathrm{R} 45$. Ce sont les relevés des zones sèches. Les relevés pouvant contribuer significativement à l'axe des abscisses négatives sont: R 5, R 6, R 8, R 11, R 16, R 17, R 19, R 20, R 21, R 28, R 29, R 31, R 32, $\mathrm{R} 33$ et $\mathrm{R} 38$. Ces relevés correspondent à ceux des zones humides.

La Figure 4 représente le diagamme de l'AFC sur la matrice 45 relevés x 100 espèces. Sur cette figure, l'axe 1 oppose deux groupes: sur l'axe des abscisses positives se trouvent les relevés du groupe 1. Les espèces associées à ces relevés et ayant une contribution significative sont celles des groupements 4 et 5. Il s'agit de: Merremia pinnata; Digitaria sp, Acacia tortilis subsp. raddiana, Boerhavia erecta, Datura inoxia, Ipomoea coptica, 
Mitracarpus hirtus, Drimia indica, Balanites aegyptiaca, Tragus racemosa, Aerva javanica, Crotalaria podocarpa, Commelina forskaolii, Commiphora africana, Adansonia digitata, Pennisetum violaceum, Indigofera pilosa, Acacia senegal, Schoenefeldia gracilis, Monsonia senegalensis, Blepharis linearifolia, Dipcadi viride, Cleome tenela, Gisekia pharnaceoides, Sclerocarya birrea, Combretum acculeatum. Ce groupe est caractéristique des zones les plus sèches situées sur les dunes et les plaines. Les relevés du groupe 2 se trouvent sur l'axe des abscisses négatives. Les espèces associées à ces relevés et ayant une contribution significative sont celles des groupements 1,2 et 3 . Il s'agit de: Cissus palmatifida, Echinochloa colona, Avicennia germinans, Leptochloa fusca, Salicornia senegalensis, Cressa cretica, Sporobolus virginicus, Asparagus flagellaris, Stylochaeton hypogaeus, Sporobolus spicatus, Tetraena gaetula subsp. waterlotii, Cyperus jeminicus, Cucumis melo, Indigofera tinctoria, Sesbania pachycarpa, Tamarix senegalensis, Sesuvium portulacastrum, Cissus quadrangularis, Salsola baryosma, Leptadenia lanceolata et Setaria verticilata. Ces espèces se rencontrent dans les zones les plus humides. Ainsi, l'axe 1 pourrait être considéré comme un gradient d'humidité.

L'axe 2 sépare les relevés des zones les plus sèches en deux groupes. Au niveau des abscisses positives de l'axe 2 sont présents les relevés suivants: R 3, R 10 R 13, R 26, R 35, R 36 R 37, R 39, R 41, R 42, et R 44. Il s'agit des relevés du groupement 4 . Ces relevés sont associés aux espèces suivantes: Prosopis glandulosa Acacia nilotica, Cucumis ficifolius, Commelina benghalensis, Cocculus pendulus, Indigofera colutea, Ipomea dichroa, Boscia senegalensis, Opuntia tuna, Solanum forskaolii, Salvadora persica, Dicliptera paniculata, Heliotropium bacciferum, Commicarpus helenae, Euphorbia balsamifera, Eragrostis tremula, Grewia tenax, Amaranthus hybridus, Brachiaria ramosa et Commiphora africana. Ces espèces sont rencontrées dans des zones où l'altitude est plus élevée (dunes). Au niveau des abscisses négatives de l'axe 2 , sont présents les relevés du groupement 5 . Ce sont les relevés R 36, R 16, R 43, R 34, R 24, R 12, R 45, R 25, R 4, R 40, R 2, R 1 et R 30. Ces relevés sont associés essentiellement à des thérophytes. Il s'agit de: Alysicarpus ovalifolius, Cenchrus biflorus, Indigofera aspera, Eragrostis ciliaris, Polycarpon prostratum, Lippia chevalieri, Dactyloctenium aegyptium, Limeum diffusum, Tribulus terrestris, Zornia glochidiata, Leersia hexandra, Indigofera senegalensis, Aristida sieberiana, Phyllantus amarus, Cyamopsis senegalensis, Corchorus tridens, Senna italica, Jacquemontia tamnifolia, Pavonia zeylanica, Tephrosia gracilipes, Digitaria perrottetii, Limeum viscosum, Polycarpea linearifolia. Ces espèces sont rencontrées au niveau des plaines basses. Ainsi, l'axe 2 pourrait être considéré comme un gradient topographique et un gradient de thérophytisation croissant des abscisses positifs aux abscisses négatifs.

L'analyse des résultats de l'AFC permet de distinguer cinq groupements au niveau de la Réserve Spéciale de Faune de Gueumbeul.

Le groupement 1: c'est un groupement à Salicornia senegalensis. Il se développe sur un sol argilo-sableux humide. Il comprend les relevés des zones les plus humides: R8, R28, R32 et R38. Ces relevés sont associés aux espèces suivantes: Salicornia senegalensis, Avicennia germinans, Echinochloa colona, Cressa cretica, Sporobolus virginicus et Leptochloa fusca.

Le groupement 2: c'est un groupement à Tetraena gaetula subsp. waterlotii qui s'établit sur un sol sableux parfois humide parfois sec et dur. Il comporte, en dehors de Tetraena gaetula subsp. waterlotii, les espèces suivantes: Asparagus flagellaris, Sporobolus spicatus, Cucumis melo, Cyperus effusus, Tephrosia gracilipes, Sesbania pachycarpa, Indigofera tinctoria, Sesuvium portulacastrum, Polycarpea linearifolia, Cyperus bulbosus, Setaria verticillata, Salsola baryosma, Tamarix senegalensis, Blutaparon vermiculare, Cissus quadrangularis et Leptadenia lanceolata. 
Le groupement 3: c'est un groupement à Prosopis glandulosa qui s'installe souvent sur un sol sableux et rarement sur un sol argileux ou argilo-sableux. Les autres espèces du groupement sont: Acacia nilotica, Cucumis ficifolius, Commelina benghalensis, Cocculus pendulus, Indigofera colutea, Ipomoea dichroa et Boscia senegalensis.

Le groupement 4: c'est un groupement à Opuntia tuna qui s'établit sur un sol sableux. Il comprend en dehors d'Opuntia tuna, Solanum forsskaolii, Salvadora persica, Dicliptera paniculata, Heliotropium bacciferum, Commicarpus helenae, Euphorbia balsamifera, Eragrostis tremula, Grewia tenax, Amaranthus hybridus, Brachiaria ramosa et Commiphora africana.

Le groupement 5: c'est le groupement à Acacia tortilis var. raddiana. Il se développe sur un sol sableux. Les espèces qui forment ce groupement en dehors d'Acacia tortilis var. raddiana sont: Datura inoxia, Dactyloctenium aegyptium, Aristida sieberiana, Corchorus tridens, Lippia chevalieri, Cenchrus biflorus, Eragrostis ciliaris, Tribulus terrestris, Indigofera aspera, Gisekia pharnaceoides, Crotalaria podocarpa, Balanites aegyptiaca, Cleome tenella, Leersia hexandra, Cyamopsis senegalensis, Limeum diffusum, Zornia glochidiata, Zaleya pentandra, Polycarpon prostratum, Phyllanthus amarus, Mitracarpus hirtus, Indigofera pilosa, Pennisetum violaceum, Adansonia digitata, Acacia senegal, Enteropogon prieuri, Achyrantes aspera, Merremia tridentata et Polycarpea corymbosa.

Tableau 2: Liste des espèces recensées avec des indications sur leur type biologique (TB), leur répartition géographique $(\mathrm{RG})$, l'abondance dominance moyenne ( $\mathrm{AD} / \mathrm{MOY})$, leur fréquence (FR) et l'indice de fréquence (IF).

\begin{tabular}{llccccc}
\hline Familles & Espèces & TB & RG & AD/MOY & FR & IF \\
\hline Acanthaceae (D) & Blepharis linariifolia Pers. & T & As & 0,1 & 0,02 & I \\
& Dicliptera paniculata (Forssk.) I. Darbysh. & T & Ase & 2,13 & 0,09 & I \\
\hline Aizoaceae (D) & Gisekia pharnaceoides L. & T & Mas & 0,36 & 0,11 & I \\
& Limeum diffusum (J. Gay) Schinz. & T & Af & 0,55 & 0,04 & I \\
& Limeum viscosum (J.Gay) Fenzl. & T & Af & 0,5 & 0,02 & I \\
& Sesuvium portulacastrum L. & C & Asm & 0,48 & 0,11 & I \\
& Zaleya pentandra (L.) C. Jeffrey & C & As & 0,55 & 0,04 & I \\
\hline Amarantaceae (D) & Achyranthes aspera L. & T & Cosm & 1,81 & 0,33 & II \\
& Aerva javanica (Burm. f.) Juss. ex Schult. & C & As & 0,31 & 0,18 & I \\
& Amaranthus hybridus L. & T & Pt & 0,1 & 0,02 & I \\
& Blutaparon vermiculare (L.) Mears & C & Am & 0,55 & 0,04 & I \\
& Caroxylon imbricatum (For.) Akh. \& Roal. & C & As & 0,81 & 0,2 & II \\
\hline Anacardiaceae (D) & Sclerocarya birrea (A. Rich.) Hochst. & P & Af & 0,1 & 0,02 & I \\
Araceae (M) & Stylochaeton hypogaeus Lepr. & G & Af & 0,5 & 0,02 & I \\
Apocynaceae - & & & & & & \\
Asclepioideae (D) & Leptadenia lanceolata (Poir.) Goyder & P & Af & 0,39 & 0,2 & II \\
Asparagaceae (M) & Asparagus flagellaris (Kunth.) Baker & P & Af & 0,1 & 0,02 & I \\
Asteraceae (D) & Baccharoides adoensis (Sch. Bip.) H. Rob. & C & Af & 0,1 & 0,02 & I \\
Avicenniaceae (D) & Avicennia germinans (L.) L. & P & Af & 1,2 & 0,07 & I \\
Balanitaceae (D) & Balanites aegyptiaca (L.) Delile. & P & As & 1,01 & 0,18 & I \\
Boraginaceae (D) & Heliotropium bacciferum Forsk. & T & As & 0,55 & 0,04 & I
\end{tabular}




\begin{tabular}{|c|c|c|c|c|c|c|}
\hline Burseraceae (D) & Commiphora africana (A. Rich.) Engl. & $\mathrm{P}$ & Af & 0,36 & 0,22 & II \\
\hline Cactaceae (D) & Opuntia tuna (L.) Mill. & $\mathrm{P}$ & Am & 2,73 & 0,33 & II \\
\hline Capparaceae (D) & Boscia senegalensis (Pers.) Lam. & $\mathrm{P}$ & Af & 0,1 & 0,04 & $\mathrm{I}$ \\
\hline & Cleome tenella L. f. & $\mathrm{T}$ & Mas & 0,1 & 0,09 & $\mathrm{I}$ \\
\hline Caryophyllaceae (D) & Polycarpaea corymbosa (L.) Lam. & $\mathrm{T}$ & Asm & 0,3 & 0,04 & I \\
\hline & Polycarpaea linearifolia (DC.) DC. & $\mathrm{T}$ & Af & 0,3 & 0,04 & I \\
\hline & Polycarpon prostratum (For.) Asch. \& Sch. & $\mathrm{H}$ & Ame & 0,1 & 0,02 & I \\
\hline Chenopodiaceae (D) & Salicornia senegalensis A.Chev. & $\mathrm{T}$ & Masue & 2,59 & 0,22 & II \\
\hline Combretaceae (D) & Combretum aculeatum Vent. & $\mathrm{P}$ & Af & 0,1 & 0,02 & I \\
\hline Commelinaceae (M) & Commelina benghalensis L. & $\mathrm{T}$ & As & 0,5 & 0,02 & $\mathrm{I}$ \\
\hline & Commelina forskaolii Vahl. & $\mathrm{T}$ & Mas & 1,59 & 0,4 & III \\
\hline Convolvulaceae (D) & Cressa cretica $\mathrm{L}$. & $\mathrm{T}$ & M & 1,17 & 0,07 & I \\
\hline & Ipomoea coptica (L.) Roth & $\mathrm{T}$ & Asu & 0,55 & 0,04 & I \\
\hline & Ipomoea dichroa Choisy. & $\mathrm{T}$ & Asu & 1,05 & 0,04 & I \\
\hline & Jacquemontia tamnifolia (L.) Griseb. & $\mathrm{T}$ & $\mathrm{Am}$ & 1 & 0,02 & I \\
\hline & Merremia pinnata (Hochst.) Hall. & $\mathrm{T}$ & Af & 1,47 & 0,31 & II \\
\hline & Merremia tridentata ( L.) Hallier f. & $\mathrm{T}$ & Af & 0,8 & 0,11 & I \\
\hline Cucurbitaceae (D) & Cucumis ficifolius A. Rich. & $\mathrm{T}$ & As & 0,34 & 0,11 & $\mathrm{I}$ \\
\hline & Cucumis melo subsp. agrestis (Naudin) & & & & & \\
\hline & Pangalo & $\mathrm{T}$ & As & 0,4 & 0,07 & I \\
\hline & Cucumis metiluferus E. Mey. & $\mathrm{T}$ & Af & 0,1 & 0,02 & I \\
\hline Cyperaceae $(\mathrm{M})$ & Cyperus bulbosus Vahl. & G & Asu & 3,33 & 0,07 & I \\
\hline & Cyperus jeminicus Rottb. & $\mathrm{H}$ & As & 1,33 & 0,07 & I \\
\hline Euphorbiaceae (D) & Euphorbia balsamifera Aiton. & $\mathrm{P}$ & Af & 1,28 & 0,29 & II \\
\hline Fabaceae - & & & & & & \\
\hline Caesalpinoideae (D) & Senna italica Mill. & $\mathrm{T}$ & As & 0,1 & 0,07 & I \\
\hline $\begin{array}{l}\text { Fabaceae - Faboideae } \\
\text { (D) }\end{array}$ & $\begin{array}{l}\text { Alysicarpus ovalifolius (Sch. et Thonn.) J. } \\
\text { Léonard }\end{array}$ & $\mathrm{T}$ & $\mathrm{Pt}$ & 0,41 & 0,27 & II \\
\hline & Crotalaria podocarpa DC. & $\mathrm{T}$ & $\mathrm{M}$ & 1,44 & 0,11 & I \\
\hline & Cyamopsis senegalensis Guill et Perr. & $\mathrm{T}$ & As & 0,5 & 0,02 & I \\
\hline & Indigofera aspera Perr. & $\mathrm{T}$ & Af & 0,53 & 0,27 & II \\
\hline & Indigofera colutea (Burm. f.) Merr. & $\mathrm{T}$ & Asu & 1 & 0,02 & I \\
\hline & Indigofera pilosa Poir. & $\mathrm{T}$ & Af & 0,53 & 0,18 & I \\
\hline & Indigofera senegalensis Lam. & $\mathrm{T}$ & Af & 1 & 0,02 & I \\
\hline & Indigofera tinctoria $\mathrm{L}$. & $\mathrm{C}$ & As & 1 & 0,07 & I \\
\hline & Sesbania pachycarpa DC. & $\mathrm{T}$ & Asu & 0,7 & 0,04 & I \\
\hline & Tephrosia gracilipes Guill. et Perr. & $\mathrm{T}$ & Af & 0,1 & 0,02 & I \\
\hline & Zornia glochidiata Reichb. & $\mathrm{T}$ & $\mathrm{Pt}$ & 0,1 & 0,02 & I \\
\hline Fabaceae - & Acacia nilotica (L.) Willd. & $\mathrm{P}$ & As & 0,64 & 0,11 & I \\
\hline Mimosoideae & Acacia tortilis (Forssk.) Hayne subsp. & & & & & \\
\hline (D) & raddiana (Savi) Brenan. & $\mathrm{P}$ & As & 2,36 & 0,36 & II \\
\hline & $\begin{array}{l}\text { Acacia senegal (L.) Willd. } \\
\text {. }\end{array}$ & $\mathrm{P}$ & As & 1,6 & 0,16 & I \\
\hline & Prosopis glandulosa Torr. & $\mathrm{P}$ & $\mathrm{Pt}$ & 1,86 & 0,42 & III \\
\hline Geraniaceae (D) & Monsonia senegalensis Guill. \& Perr. & $\mathrm{T}$ & As & 1,7 & 0,07 & I \\
\hline Hyacinthaceae (M) & Dipcadi viride (L.) Moench & $\mathrm{G}$ & Af & 0,36 & 0,18 & I \\
\hline & Drimia indica (Roxb.) Jessop & $\mathrm{G}$ & As & 0,1 & 0,04 & I \\
\hline Malvaceae - & Adansonia digitata $\mathrm{L}$. & $\mathrm{P}$ & $\mathrm{M}$ & 0,1 & 0,04 & $\mathrm{I}$ \\
\hline
\end{tabular}




\begin{tabular}{|c|c|c|c|c|c|c|}
\hline Bombacoideae (D) & & & & & & \\
\hline Malvaceae - & & & & & & \\
\hline Malvoideae (D) & Pavonia zeylanica Cav. & $\mathrm{T}$ & AS & 0,68 & 0,09 & I \\
\hline Malvaceae - Tilioideae & Corchorus tridens $\mathrm{L}$. & $\mathrm{T}$ & Asu & 0,52 & 0,24 & II \\
\hline (D) & Grewia tenax (Forssk.) Fiori. & $\mathrm{P}$ & As & 0,82 & 0,11 & I \\
\hline Nyctaginaceae (D) & Boerhavia erecta $\mathrm{L}$. & $\mathrm{C}$ & $\mathrm{Pt}$ & 0,4 & 0,07 & $\mathrm{I}$ \\
\hline & Commicarpus helenae (Schult.) Meikle & $\mathrm{C}$ & Ase & 0,36 & 0,22 & II \\
\hline Pedaliaceae (D) & Sesamum alatum Thonn. & $\mathrm{T}$ & Af & 0,1 & 0,02 & $\mathrm{I}$ \\
\hline Phyllanthaceae & Phyllantus amarus Sch. \& Thonn. & $\mathrm{T}$ & $\mathrm{Pt}$ & 0,23 & 0,07 & I \\
\hline Poaceae (M) & Aristida sieberiana Trin. & $\mathrm{H}$ & As & 0,73 & 0,36 & II \\
\hline & Brachiaria ramosa (L.) Stapf. & $\mathrm{T}$ & As & 0,68 & 0,13 & I \\
\hline & Cenchrus biflorus Roxb. & $\mathrm{T}$ & As & 0,23 & 0,33 & II \\
\hline & Dactyloctenium aegyptium (L.) Willd. & $\mathrm{T}$ & $\mathrm{Pt}$ & 0,3 & 0,22 & II \\
\hline & Digitaria perrottetii (Kunth) Stapf & $\mathrm{T}$ & M & 1 & 0,02 & I \\
\hline & Digitaria sp. (Forssk.) P. Beauv. & $\mathrm{T}$ & AmAsu & 0,28 & 0,2 & II \\
\hline & Leptochloa fusca (L.) Kunth. & $\mathrm{H}$ & Asu & 3,58 & 0,13 & I \\
\hline & Echinochloa colona (L.) Link. & $\mathrm{T}$ & $\mathrm{Pt}$ & 0,1 & 0,02 & I \\
\hline & Enteropogon prieurii (Kunth) Clayton. & $\mathrm{T}$ & Af & 0,68 & 0,38 & II \\
\hline & Eragrostis ciliaris (L.) R. Br. & $\mathrm{T}$ & $\mathrm{Pt}$ & 0,2 & 0,18 & I \\
\hline & Eragrostis tremula (Lam.) Hochst. & $\mathrm{T}$ & As & 0,1 & 0,04 & I \\
\hline & Leersia hexandra Sw. & G & $\mathrm{Pt}$ & 0,44 & 0,11 & I \\
\hline & Pennisetum violaceum (Lam.) Rich. & $\mathrm{T}$ & Asu & 0,87 & 0,07 & $\mathrm{I}$ \\
\hline & Schoenefeldia gracilis Kunth. & $\mathrm{T}$ & As & 0,1 & 0,02 & I \\
\hline & Setaria verticillata (L.) P. Beauv. & $\mathrm{T}$ & $\mathrm{Pt}$ & 0,91 & 0,04 & I \\
\hline & Sporobolus spicatus Kunth. & $\mathrm{H}$ & As & 1,74 & 0,11 & I \\
\hline & Sporobolus virginicus (L.) Kunth. & G & $\mathrm{Pt}$ & 1 & 0,02 & I \\
\hline & Tragus racemosus (L.) All & $\mathrm{T}$ & As & 0,37 & 0,07 & $\mathrm{I}$ \\
\hline Polygalaceae (D) & Polygala baikiei subsp. pobeguinii (A. Chev. & & & & & \\
\hline & \& Jacq.Fél.) Paiva & $\mathrm{T}$ & Af & 1,05 & 0,04 & I \\
\hline Rubiaceae (D) & Mitracarpus hirtus (L.) DC. & $\mathrm{T}$ & $\mathrm{Ae}$ & 0,53 & 0,07 & I \\
\hline Salvadoraceae (D) & Salvadora persica $\mathrm{L}$. & $\mathrm{p}$ & As & 0,63 & 0,24 & II \\
\hline Solanaceae (D) & Datura inoxia Mill. & $\mathrm{T}$ & $\mathrm{Ae}$ & 1,25 & 0,04 & $\mathrm{I}$ \\
\hline & Solanum forsskalii Dunal & $\mathrm{C}$ & As & 0,5 & 0,02 & I \\
\hline Tamaricaceae (D) & Tamarix senegalensis DC. & $\mathrm{P}$ & $\mathrm{Pt}$ & 1,26 & 0,16 & I \\
\hline Verbenaceae (D) & Lippia chevalieri Moldenk. & $\mathrm{T}$ & Af & 0,78 & 0,18 & $\mathrm{I}$ \\
\hline Vitaceae (D) & Cissus palmatifida (Baker.) Planch. & $\mathrm{H}$ & Af & 0,1 & 0,02 & $\mathrm{I}$ \\
\hline & Cissus quadrangularis L. & $\mathrm{P}$ & As & 0,1 & 0,04 & $\mathrm{I}$ \\
\hline Zygophyllaceae (D) & Tribulus terrestris $\mathrm{L}$. & $\mathrm{T}$ & Cosm & 0,27 & 0,16 & $\mathrm{I}$ \\
\hline & Tetraena gaetula subsp. waterlotii (Maire) & & & & & \\
\hline & Beier \& Thulin & $\mathrm{C}$ & Af & 1,19 & 0,22 & II \\
\hline
\end{tabular}


Tableau 3 : Répartition par famille des espèces de la flore de la RSFG.

\begin{tabular}{|c|c|c|}
\hline \multicolumn{3}{|c|}{ Flore de la réserve spéciale de faune de Gueumbeul } \\
\hline Familles & Nombre d'espèces & Pourcentage Familles \\
\hline Poaceae (M) & 18 & $18 \%$ \\
\hline Fabaceae (D) & 17 & $17 \%$ \\
\hline Convolvulaceae (D) & 6 & $6 \%$ \\
\hline Aizoaceae (D) & 5 & $5 \%$ \\
\hline Amaranthaceae (D) & 5 & $5 \%$ \\
\hline Caryophyllaceae (D) & 3 & $3 \%$ \\
\hline Cucurbitaceae (D) & 3 & $3 \%$ \\
\hline Malvaceae & 3 & $3 \%$ \\
\hline Acanthaceae (D) & 2 & $2 \%$ \\
\hline Ampelidaceae (D) & 2 & $2 \%$ \\
\hline Capparaceae (D) & 2 & $2 \%$ \\
\hline Commelinaceae (M) & 2 & $2 \%$ \\
\hline Cyperaceae (M) & 2 & $2 \%$ \\
\hline Hyacinthaceae (M) & 2 & $2 \%$ \\
\hline Nyctaginaceae (D) & 2 & $2 \%$ \\
\hline Solanaceae (D) & 2 & $2 \%$ \\
\hline Zygophyllaceae (D) & 2 & $2 \%$ \\
\hline Anacardiaceae (D) & 1 & $1 \%$ \\
\hline Apocynaceae & 1 & $1 \%$ \\
\hline Araceae( M) & 1 & $1 \%$ \\
\hline Asparagaceae (M) & 1 & $1 \%$ \\
\hline Asteraceae (D) & 1 & $1 \%$ \\
\hline Avicenniaceae (D) & 1 & $1 \%$ \\
\hline Balanitaceae (D) & 1 & $1 \%$ \\
\hline Boraginaceae(D) & 1 & $1 \%$ \\
\hline Burseraceae (D) & 1 & $1 \%$ \\
\hline Cactaceae D) & 1 & $1 \%$ \\
\hline Chenopodiaceae (D) & 1 & $1 \%$ \\
\hline Combretaceae (D) & 1 & $1 \%$ \\
\hline Euphorbiaceae (D) & 1 & $1 \%$ \\
\hline Geraniaceae (D) & 1 & $1 \%$ \\
\hline Pedaliaceae (D) & 1 & $1 \%$ \\
\hline Phyllantaceae & 1 & $1 \%$ \\
\hline Piperaceae (D) & 1 & $1 \%$ \\
\hline Polygalaceae (D) & 1 & $1 \%$ \\
\hline Rubiaceae (D) & 1 & $1 \%$ \\
\hline Salvadoraceae (D) & 1 & $1 \%$ \\
\hline Tamaricaceae (D) & 1 & $1 \%$ \\
\hline Verbenaceae (D) & 1 & $1 \%$ \\
\hline
\end{tabular}


Tableau 4: Structure de la flore de la RSFG.

\begin{tabular}{lcccccc}
\hline \multirow{2}{*}{ Classes } & \multicolumn{2}{c}{ Familles } & \multicolumn{2}{c}{ Genres } & \multicolumn{2}{c}{ Espèces } \\
\cline { 2 - 7 } & Nombre & $\begin{array}{c}\text { Pourcentage } \\
(\boldsymbol{\%})\end{array}$ & Nombre & $\begin{array}{c}\text { Pourcentage } \\
(\boldsymbol{\%})\end{array}$ & Nombre & $\begin{array}{c}\text { Pourcentage } \\
(\boldsymbol{\%})\end{array}$ \\
\hline Dicotylédones & 34 & 87,18 & 61 & 74,39 & 74 & 74 \\
Monocotylédones & 5 & 12,82 & 21 & 25,61 & 26 & 26 \\
Total & 39 & 100 & 82 & 100 & 100 & 100 \\
\hline
\end{tabular}

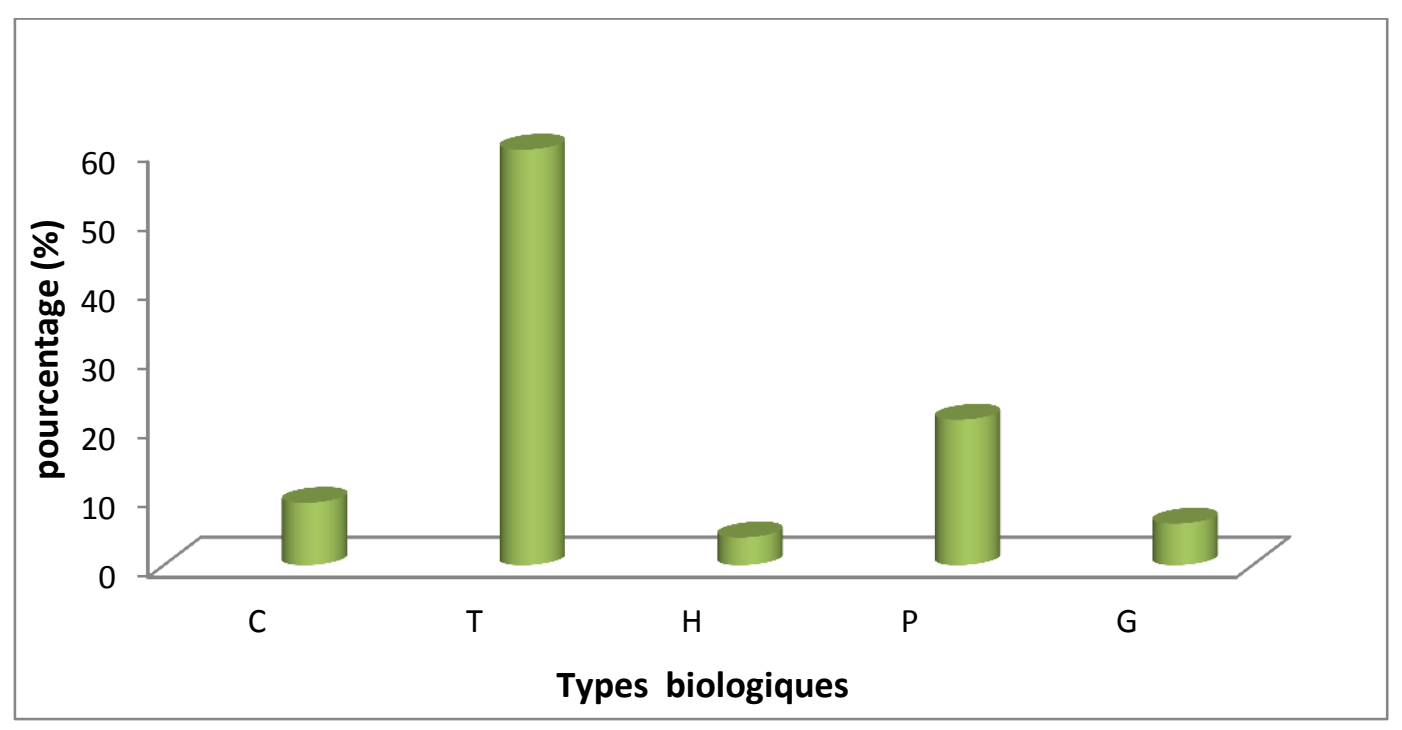

Figure 2 : Spectre biologique de la flore de la RSFG.

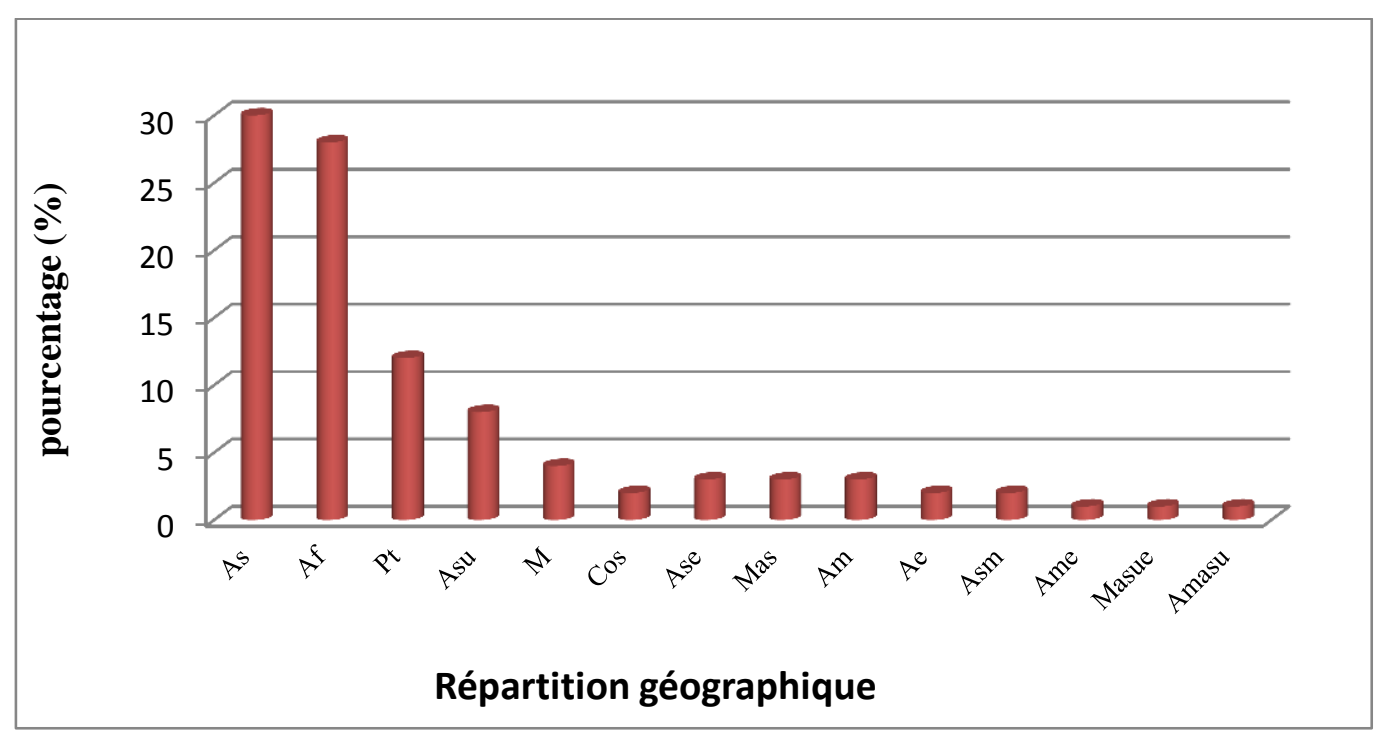

Figure 3: Répartition géographique des espèces de la flore de la RSFG. 
Tableau 5: Valeurs propres et variance expliquée par les quatre premiers axes de l'AFC.

\begin{tabular}{lcc}
\hline Axes & Valeurs propres & Pourcentage de variance \\
\hline 1 & 0,515875 & 19,676 \\
2 & 0,273886 & 10,446 \\
3 & 0,258026 & 9,8415 \\
4 & 0,229015 & 8,735 \\
\hline
\end{tabular}

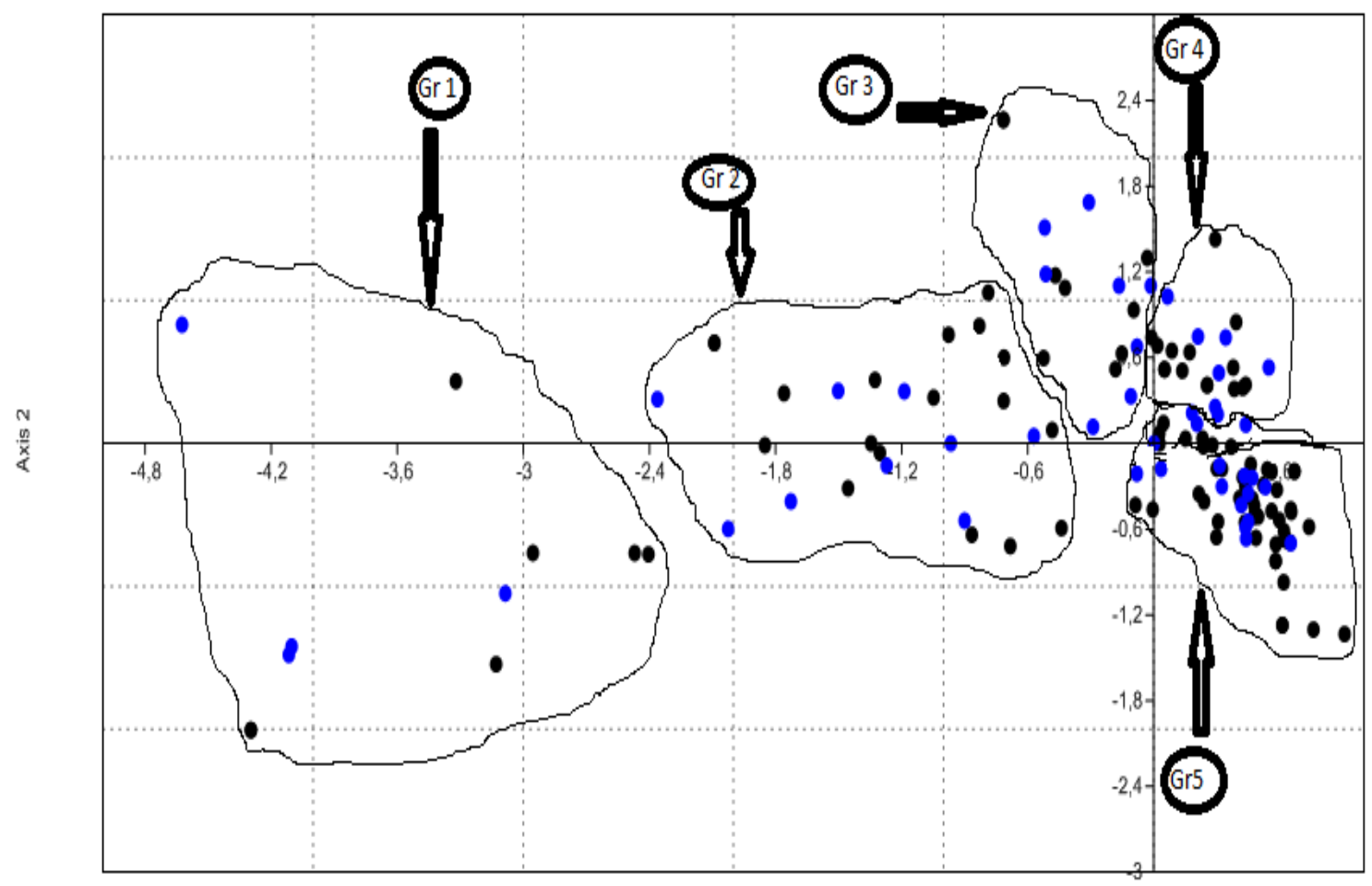

Axis 1

Figure 4 : Diagramme de l'AFC sur la matrice 45 relevés x 100 espèces.

\section{DISCUSSION}

\section{Composition spécifique}

La flore de la Réserve Spéciale de Faune de Gueumbeul comprend 100 espèces réparties dans 82 genres et 39 familles. Par comparaison aux résultats obtenus par Trochain (1940) au niveau des Niayes situé dans la même zone et constitués de 79 espèces réparties dans 56 genres et 28 familles, la flore de RSFG apparait plus riche et plus diversifiée. Cette différence pourrait s'expliquer par le statut de la RSFG qui est une zone protégée moins exposée à la dégradation du couvert végétal par l'homme et le bétail. En revanche, la flore de la RSFG est moins diversifiée que celle du Parc National des Oiseaux de Djoudj (Noba et al., 2010) qui est composée de 132 espèces appartenant à 99 genres et 48 familles. Ceci s'expliquerait par la grande diversité des écosystèmes rencontrés dans le Parc caractéristiques de milieux terrestres, saharien et sahélien et de milieux semi aquatique et aquatique d'eau douce et saumâtre. 


\section{Le spectre taxonomique}

La plupart des familles sont principalement constituées d'herbacées qui sont majoritairement des espèces annuelles. Les espèces ligneuses sont constituées essentiellement d'arbres épineux appartenant à la famille des Fabaceae et à la sous famille des Mimosoideae. Elles sont souvent adaptées à une faible pluviométrie. Ces caractéristiques sont celles d'une végétation tropicale de type sahélienne. Selon Hiernaux et Le Houérou (2006), les végétations sahéliennes sont dominées par des herbacées annuelles principalement des Poaceae (Graminées) associées à des ligneux épars. Cette caractéristique de la végétation sahélienne à savoir la dominance des espèces annuelles a été mise en évidence au Sénégal par Ba et Noba (2001). Les résultats obtenus par ces auteurs montrent que plus de la moitié de la flore du Sénégal est constituée d'herbacées généralement annuelles.

Les résultats obtenus sur l'importance des Poaceae et des Fabaceae dans la flore de la RSFG sont similaires à ceux obtenus au niveau de la flore vasculaire du Sénégal (Bâ et Noba, 2001) où les Poaceae $(11,40 \%)$ et les Fabaceae $(11,36 \%)$ sont également les plus représentées. Les résultats sur la consistance des familles monospécifiques sont identiques à ceux d'Illboudou (1992) sur la flore de la Réserve Spéciale Botanique de Noflaye où les familles monospécifiques représentent également la moitié des familles. Concernant les deux familles les plus diversifiées, une différence est notée entre la flore de la RSFG et celle du PNOD (Noba et al., 2010). En effet, si les deux familles les plus diversifiées au niveau de la RSFG sont les Poaceae et les Fabaceae, au niveau du Djoudj ce sont les Poaceae $(17,42 \%)$ et les Cyperaceae $(6,42 \%)$. Cette différence serait liée à la présence d'écosystèmes humides au niveau du parc national des oiseaux de Djoudj autorisant un grand développement des Cyperaceae. En effet, les deux familles les plus diversifiées sont constituées essentiellement d'herbacées qui parviennent à boucler leur cycle pendant l'hivernage donc qui trouvent des conditions favorables. Dans plus de la moitié $(56,41 \%)$ des familles de cette flore, les espèces seraient peu adaptées aux conditions climatiques et de salinité. Le reste des familles comprend des espèces qui seraient plus ou moins adaptées aux conditions climatiques et de salinité donc ayant des conditions de vie plus ou moins favorables.

Les Dicotylédones dominent largement cette flore avec $74 \%$ des espèces (soit plus des $2 / 3$ des espèces) contre $26 \%$ chez les Monocotylédones. Cette tendance se présente aussi au niveau de la flore vasculaire du Sénégal (Bâ et Noba, 2001) où les Dicotylédones viennent en tête avec $69 \%$ devant les Monocotylédones 29\%. Cependant, la RSFG compte plus de Dicotylédones (74\% contre $69 \%$ ) et moins de Monocotylédones ( $26 \%$ contre $29 \%$ ) que la flore vasculaire du Sénégal. Ce constat est aussi valable pour la flore du Parc National des Oiseaux de Djoudj (Noba et al., 2010). En effet, la flore de ce parc comprend $66,67 \%$ de Dicotylédones contre $74 \%$ pour la réserve spéciale de faune de Gueumbeul et $31,82 \%$ de Monocotylédones contre $26 \%$ pour la réserve spéciale de faune de Gueumbeul. Cela est dû au fait que les Cyperaceae et les Poaceae qui constituent l'essentiel des Monocotylédones sont plus représentées dans la flore vasculaire du Sénégal $(7,52 \%)$ et dans la flore du PNOD $(6,82 \%)$ que dans la flore de la RSFG (2\%).

\section{Spectre biologique}

Le spectre biologique de la flore de la RSFG est dominé par les thérophytes qui constituent $60 \%$ des espèces. Ces résultats sont similaires à ceux obtenus par Noba et al., (2010) où les thérophytes sont représentés par $50 \%$ des espèces. Ces Thérophytes sont constitués essentiellement de Poaceae, de Fabaceae, de Convolvulaceae et de Cucurbitaceae. Cependant les Poaceae et les Fabaceae constituent l'essentiel de ces Thérophytes.

La dominance des Thérophytes serait liée à leur adaptation à la pluviométrie relativement faible de la zone. Leur capacité à boucler leur cycle en quelques semaines (Noba et al., 2010) constitue un atout majeur qui explique aussi leur dominance. Les 
espèces pérennes représentent $40 \%$ de la flore. Elles sont composées de Phanérophytes appartenant principalement à la famille des Fabaceae-Mimosoideae (Acacia nilotica, A. tortilis var. raddiana, A. senegal, Prosopis glandulosa), des Cactaceae, des Rhizophoraceae, des Tamaricaceae, des Salvadoraceae etc, de Chaméphytes, d'Hémicryptophytes et de Géophytes. L'importance relative des espèces pérennes pourrait s'expliquer par plusieurs facteurs dont l'adaptation au climat et à la salinisation, la protection du site, la dissémination des semences par les animaux et le reboisement.

\section{Répartition géographique}

La flore de la RSFG est plus riche en espèces afro-asiatique et africaines comparée à la flore adventice des niayes (Sarr et al., 2007) et à la flore du PNOD (Noba et al., 2010). En effet $30 \%$ des espèces recensées sont afro-asiatiques et $28 \%$ sont africaines. Par contre, dans la flore adventice des cultures d'oignon des Niayes et du PNOD, les espèces afro-asiatiques représentent réspectivement $4,6 \%$ et $21,21 \%$, les espèces africaines $22,8 \%$ et $17,42 \%$. Cependant, elle compte moins d'espèces d'affinité pantropicale que ces dernières $(12 \%$ contre $27,5 \%$ et $31,82 \%$ respectivement pour la flore adventice des cultures d'oignon des Niayes et le PNOD). Le nombre d'affinités biogéographiques élevé serait lié à l'introduction d'espèces par divers agents de transport dont les plus importants pourraient être les oiseaux migrateurs. En effet, la RSFG est un site de passage et de nichage d'oiseaux migrateurs (UICN/PACO, 2012).

\section{Identification des indices de fréquence}

Les espèces rares ou accidentelles dominent largement la flore de la RSFG. Elles sont suivies des espèces accessoires puis des espèces fréquentes. Ces résultats sont similaires à ceux obtenus au niveau de la flore des formations végétales de Massenya au Tchad (Melow et al., 2015) où les espèces rares sont dominantes et les espèces fréquentes minoritaires. Par contre, ces résultats sont différents de ceux de la flore des deux mares dans le parc national du Niokolo koba (Diop et Noba., 2015) où les espèces abondantes et constantes sont les plus présentes. L'importance relative des espèces rares ou accidentelles et des espèces accessoires laisse penser qu'il s'agit de plusieurs milieux plus ou moins diversifiés et plurispécifiques, stables et naturels par endroits parce que protégés mais subissant la rigueur de l'aridité du climat et par endroit la salinité. Ils sont différents des mares du PNNK qui sont des milieux perturbées envahis par des plantes envahissantes qui se sont proliférées. La proportion relativement faible d'espèces fréquentes est liée à l'appartenance du site à la zone sahélienne. L'espèce Opuntia tuna dont l'invasion constitue une menace pour la Réserve Spéciale de Faune de Gueumbeul est accessoire dans cette réserve. Cette espèce a été introduite dans la Réserve Spéciale de Faune de Gueumbeul vers le milieu des années 90 pour servir de clôture (WWF WAMER, 2011). En effet, on distingue deux populations d'Opuntia tuna dans la réserve. La première population est située en amont et la deuxième en aval de la cuvette. Les individus constituant la première population forment des haies vives de tailles différentes. Par contre, la deuxième population est représentée par quelques individus. Ce statut est pour le moment différent de celui de Mimosa pigra dans les mares du Parc National du Niokolo koba (Gueye et Noba, 2015) qui est une espèce constante en bordure et à l'intérieure de la mare de Nianaka et fréquente autour de celle-ci. Il faut espérer que cette espèce ne change de statut en proliférant audelà des zones infestées. Il semble que des travaux de dessouchage manuel réguliers d'Opuntia tuna, lents, pénibles mais efficaces diminuent son invasion (Faye, 2011).

\section{Identification des groupements}

$\mathrm{Au}$ niveau de la RSFG, cinq groupements ont été identifiés. Parmi eux, deux groupements ont été déjà décrits. Il s'agit du groupement à Salicornia senegalensis décrit par Trochain (1940) et 
Raynal (1963) et celui à Acacia tortilis subsp.raddiana Trochain (1940).

Le groupement à Salicornia senegalensis se développe sur des sols argileux humides et salés près de la cuvette de Gueumbeul. Ce groupement est aussi pauvre que son homologue décrit par Raynal (1963) aux environs de Kayar et celui décrit par Trochain (1940). Cependant, le groupement décrit par Trochain est légèrement plus riche que les deux autres. Les espèces communes entre le groupement à Salicornia senegalensis décrit à Gueumbeul et ses deux homologues décrits par Raynal (1963) et Trochain (1940) sont: Cressa cretica et Salicornia senegalensis. Le groupement à Salicornia senegalensis constitue avec le groupement à Tetraena gaetula subsp.waterlotii les deux groupements halophytes au niveau de la réserve. Dans ce groupement, une seule espèce est à affinité biogéographique africaine. Il s'agit d'Avicennia germinans. Cela s'expliquerait par la fréquentation du site par des oiseaux migrateurs.

Le groupement à Tetraena gaetula subsp.waterlotii ou groupement 2 est souvent rencontré à côté du groupement à salicornia senegalensis. Tetraena gaetula subsp.waterlotii est une espèce buissonnante des milieux salés. Parmi les espèces qui accompagnent Tetraena gaetula subsp.waterlotii dans ce groupement figurent beaucoup d'halophytes comme Salsola baryosma, Tamarix senegalensis, Sesuvium portulacastrum. Ce qui témoigne du degré de salinisation du sol qui abrite le groupement. Tetraena gaetula subsp.waterlotii figure dans un groupement végétal au niveau du parc national du banc d'arguin sous le nom de Zygophyllum waterlotii (Correra, 2006). Il partage ce groupement végétal avec des espèces comme Euphorbia balsamifera, Nucularia perinni, Traganum nudatum, Salsola sp, Salvadora persica et Nitraria retusa.

Le groupement 3 est le groupement à Prosopis glandulosa. L'espèce Prosopis glandulosa est une plante originaire d'Amérique introduite au Sénégal. Cependant, elle s'adapte bien aux conditions climatiques et édaphiques. Elle est dotée d'un grand pouvoir de régénération. L'espèce est très prisée par la population surtout par les femmes grâce à la qualité de son bois. Elle a été signalée dans le groupement à Acacia tortilis subsp.raddiana décrit par Ndong et al. (2015) au Ferlo. Ce groupement est floristiquement pauvre. En effet, il est fréquent de trouver sous Prosopis glandulosa quelques individus chétifs. Ce phénomène serait dû à un effet allélopathique de la plante, souligné par Dave et Bandari (2013), et qui pourrait expliquer en partie la pauvreté du groupement.

Le groupement 4 est un groupement à Opuntia tuna. Opuntia tuna est une phanérophyte charnue vivace de 1 à $1,5 \mathrm{~m}$ qui forme des buissons impénétrables. Le genre Opuntia est originaire des zones tropicales du continent nord-américain, en particulier du Mexique, où l'on a retrouvé des graines fossiles datant du septième millénaire avant $\mathrm{J}$ $\mathrm{C}$ et indiquant l'utilisation alimentaire de l'espèce à l'époque préhistorique (Mulas et Mulas, 2004). Floristiquement, le groupement est relativement pauvre. Cette pauvreté du groupement peut s'expliquer par son caractère d'espèce envahissante. En effet, la plante est dotée d'un grand pouvoir de multiplication soit par les graines ou de manière végétative. Elle possède aussi un grand pouvoir de régénération. Même brulée et enfouie dans le sol, la plante présente des résidus qui régénèrent (Faye, 2011). En outre, c'est une plante CAM ce qui permet une moindre perte d'eau par évapotranspiration (Habibi, 2004).

Le groupement 5 est le groupement à Acacia tortilis subsp. raddiana. A. tortilis subsp. raddiana est un arbre épineux dont les feuilles et les fruits sont très appétés du bétail. Ce groupement est floristiquement plus riche que les autres groupements. Les animaux se nourrissent des gousses et des feuilles d'Acacia tortilis subsp. raddiana et en retour leurs déjections participent à la fertilisation des sols. Nous constatons qu'une espèce de ce groupement en l'occurrence Balanites aegyptiaca porte le nom de l'un des trois 
groupements identifiés par Ndong et al., (2015) dans le Ferlo. Ce groupement présente beaucoup d'espèces communes avec celui décrit par Trochain (1940). Il s'agit de Dactyloctenium aegyptium, Eragrostis ciliaris, Enteropogon prieuri, Achyrantes aspera, Cenchrus biflorus, Gisekia pharnaceoides, Eragrostis ciliaris, Indigofera aspera, Acacia senegal, Adansonia digitata et Balanites aegyptiaca. Cependant, il est floristiquement moins riche. En effet, la sécheresse et la dégradation anthropique pourraient expliquer l'appauvrissement de ce groupement.

\section{Conclusion}

L'étude floristique a montré que la flore de cette réserve est riche de 100 espèces réparties en 82 genres et 42 familles parmi lesquelles, les Poaceae, les Fabaceae, les Convolvulaceae et les Amaranthaceae sont les plus diversifiées. Les Dicotylédones dominent cette flore. Les Thérophytes constituent l'essentiel de la flore, suivis des Phanérophytes. Du point de vue de la répartition géographique, la flore de la RSFG est dominée par des espèces afro-asiatiques suivies des espèces africaines.

La végétation de la Réserve Spéciale de Faune de Gueumbeul est composée essentiellement d'espèces rares ou accidentelles. L'identification des groupements permet de distinguer deux catégories de groupements. La première catégorie comprend les groupements halophytes à proximité des dépressions. Ces groupements s'installent sur des sols argileux ou sablo-argileux. La deuxième catégorie comprend les groupements des dunes et des plaines basses qui s'érigent sur un sol sableux. Cette répartition des groupements est étroitement liée à la teneur en sel du sol.

La présence d'Opuntia tuna pourrait constituer, à terme, une menace pour l'ensemble de la Réserve si elle ne fait pas l'objet d'une attention particulière. Une gestion durable des ressources végétales de la RSFG nécessite une connaissance de la flore et de la végétation.

\section{CONFLIT D'INTERETS}

Les auteurs déclarent qu'il n'y a aucun conflit d'intérêts pour cet article.

\section{CONTRIBUTIONS DES AUTEURS}

BF a collecté, traité et analysé les données puis rédigé et corrigé l'article. $\mathrm{CB}$ et AN ont contribué à la rédaction et correction de cet article. AAC a contribué à la réactualisation des noms scientifiques. MSM a contribué au traitement des données et à la correction de l'article. KN a contribué à la rédaction et à la correction de l'article.

\section{REMERCIEMENTS}

Nos remerciements vont à l'endroit du conservateur de la RSFG et ses agents pour leurs appuis, orientations et leurs conseils.

\section{REFERENCES}

Abdourhamane H, Morou B, Rabiou H, Mahamane A. 2013. Caractéristiques floristiques, diversité et structure de la végétation ligneuse dans le centre-sud du Niger: cas du complexe des forêts classées de Dan Kada Dodo-Dan Gado. Int. J. Biol. Chem. Sci., 7(3): 1048-1068. DOI: http://dx.doi.org/10.4314/ijbcs. v7i3.13

Akpo LE, Grouzis M. 1996. Influence du couvert sur la régénération de quelques espèces ligneuses sahéliennes (Nord Sénégal, Afrique occidentale). Webbia 50(2): 247-263.

Anonym. 2009. Les plantes envahissantes une menace à la biodiversité. Vert. Inf. Env., 12: $38-46$.

Bâ AT, Noba K. 2001. Flore et biodiversité végétale au Sénégal. Scien. Chang. plan. Séch., 12(3): 149-155. http://www.jle. $\mathrm{com} / \mathrm{fr} / \mathrm{revues} / \mathrm{sec} / \mathrm{e}$ - 
docs/flore_et_biodiversite_vegetale_au_ senegal_230110/article.phtml

Bassène C, Mbaye MS, Camara AA, Kane A, Gueye M, Sylla SN, Sambou B, Noba K. 2014. La flore des systèmes agropastoraux de la Basse Casamance (Sénégal): cas de la communauté rurale de Mlomp. 2014. Int. J. Biol. Chem. Sci., 8(5): 2258-2273. DOI: http://dx.doi.org/10.4314/ijbcs.v8i5.28

Berhaut J. 1967. Flore du Sénégal (2 ${ }^{\mathrm{eme}}$ édn). Clairafrique: Dakar ; 485.

CDB. 2004. Programme de Travail sur les Aires Protégées (Programmes de travail de la CDB). CDB : Montréal ; 34.

Correra A. 2006. Dynamique de l'utilisation des ressources fourragères par les dromadaires des pasteurs nomades du parc national $\mathrm{du}$ banc d'arguin (Mauritanie). Thèse de doctorat, Muséum national d'histoire naturelle, Paris, 362.

Dave PN, Bhandari J. 2013. Prosopis juliflora: a review. Int. J. Chi., Stu., 1(3): 181-196. http://www.chemijournal.com/ vol1Issue3/sep2013/35.1.pdf

Diallo A, Guisse A, Faye MN, Saradoum G. 2009. Variabilité floristique de la végétation herbacée de la Niaye de Pikine au Sénégal. Rev. Ecol. (Terre Vie), 64: 123-133. http://documents. irevues.inist.fr/bitstream/handle/2042/55 781/RevuedEcologie_2009_64_2_123.p df? sequence $=1$

Diop A, Mandiang B. 2001. La Réserve Spéciale de Faune de Gueumbeul (R S F G). La Lettre de la Conservation, 2: 5.

Diop B, Diatta S, Charahabil M, Akpo LE. 2008. L'échantillonnage en phytoécologie: l'aire d'étude des communautés herbeuses dans la région de Dakar. J. Sci., 8(3): 25-36.

DPN. 2010. Plan de gestion de la réserve spéciale de faune de Gueumbeul (20102014). Direction des Parcs Nationaux.
Ministère de l'Environnement de la Protection de la Nature, des Bassins de rétention et des Lacs artificiels. P $66+$ annexes.

Faye B. 2011. Le cactus (Opuntia tuna) dans la flore de la réserve spéciale de faune de Gueumbeul (Saint-Louis du Sénégal): statut, utilité, modes de propagation et de gestion. Mémoire de master, Université cheikh Anta Diop de Dakar, Dakar, P. 40.

Faye E. 2010. Diagnostique partiel de la flore et de la végétation des Niayes et du Bassin arachidier au Sénégal : application de méthodes floristique, phytosociologique, ethnobotanique et cartographique. Thèse de doctorat, université libre de Bruxelles, Bruxelles, P. 266.

Gillet F. 2000. La phytosociologie synusiale intégrée: Guide méthodologique. Document 1. Laboratoire d'écologie végétale et de Phytosociologie. Université de Neuchâtel, Suisse, 68.

Gueye M, Noba K. 2015. Etude pédologique et phytosociologique de deux mares dans le parc national du Niokolo koba dans un contexte d'invasion biologique par Mimosa pigra. Int. J. Biol. Chem. Sci., 9(6): 2871-2888. DOI: http://dx.doi.org/10.4314/ijbcs.v9i6.27

Habibi Y. 2004. Contribution à l'étude morphologique, ultrastructurale et chimique de la figue de barbarie. Les polysaccharides parietaux : caractérisation et modification chimiques. Thèse de doctorat, université Joseph Fourier Grenoble 1 et université Cadi Ayyad Marrakech, 264.

Hiernaux P, Le houérou HN. 2006. Les parcours du sahel. Séch., 17(1-2): 51-71. https://www.rmportal.net/framelib/parco urs-sahel.pdf 
Hutchinson J, Dalziel JM. 1954. Flora of West Tropical Africa. (vol.1. Part 1, $2^{\text {nd }}$ edn). The White Friars Press Ltd: London.

Hutchinson J, Dalziel JM. 1963. Flora of West Tropical Africa. (vol 2, part 1, $2^{\text {nd }}$ edn), The White Friars Press Ltd: London.

Hutchinson J, Dalziel JM. 1963. Flora of West Tropical Africa. (vol 3, part 1, $2^{\text {nd }}$ edn), The White Friars Press Ltd: London.

Ilboudou. 1992. Etat et tendances évolutives de la flore et de la végétation de la réserve spéciale botanique de Noflaye (environs de Dakar-Sénégal), éléments pour un aménagement. Thèse de doctorat de troisième cycle, Université Cheikh Anta Diop de Dakar, Dakar, P. 119.

LE BRUN J. 1966. Les formes biologiques dans les végétations tropicales. Bull. Soc. Bot. France : 164-175

Lebrun JP, Stork A. 1991. Enumération des Plantes à Fleurs d'Afrique Tropicale (Vol. I). Edition Conservatoire et Jardin botanique de la ville de Genève.

Lebrun JP, Stork A. 1992. Enumération des Plantes à Fleurs d'Afrique Tropicale (Vol. II). Edition Conservatoire et Jardin botanique de la ville de Genève.

Lebrun JP, Stork A. 1995. Enumération des Plantes à Fleurs d'Afrique Tropicale (Vol. III). Edition Conservatoire et Jardin botanique de la ville de Genève.

Lebrun JP, Stork A. 1997. Enumération des Plantes à Fleurs d'Afrique Tropicale (Vol. IV). Edition Conservatoire et Jardin botanique de la ville de Genève.
Meddour R. 2011. La méthode phytosociologique sigmatiste ou Braunblanqueto-tüxenienne. Université Mouloud Mammeri de Tizi Ouzou, Algérie, P 40.

Melow S, Mbayngone E, Bechir AB, Ratna N, Mapongmetsem PM. 2015. Caracteristique floristique et écologique des formations végétales de Massenya au Tchad (Afrique centrale). J. Ani and Pla. Sci., $\quad$ 25(1): 3799-3813. http://www.m.elewa.org/JAPS/2015/25.1 12.\%20elisee.pdf

Mulas M, Mulas G. 2004. Potentialités d'utilisation stratégique des plantes des genres Atriplex et Opuntia dans la lutte contre la désertification. Groupe de recherche sur la désertification, Université des études de Sassari, Italie, P 112.

Ndong AT, Ndiaye O, Sagna MB, Diallo A, Galop D, Guissé A. 2015. Caractérisation de la végétation ligneuse sahélienne du Sénégal: cas du Ferlo. Int. J. Biol. Chim. Sci., 9(6): 2582-2594. DOI: http://dx.doi.org/10.4314/ijbcs.v9i6.6

Noba K, Mbaye MS, Coundoul M, Kane A, Hane PD, Bâ N, Mbaye N, Faye MN, Bâ AT. 2010. La flore du parc national des oiseaux de Djoudj. Une zone humide du Sénégal. Scien. Chang. Plan. Sech., 21(1): 71-78. DOI: 10.1684/sec.2009.0208. 